\title{
Thymoquinone Suppresses Cellular Proliferation, Inhibits VEGF Production and Obstructs Tumor Progression and Invasion in the Rat Model of DMH-Induced Colon Carcinogenesis
}

\author{
Wasfi Asfour $^{1 *}$, Sawsan Almadi ${ }^{1}$, Lina Haffar $^{2}$ \\ ${ }^{1}$ Department of Pharmacology and Toxicology, College of Pharmacy, Damascus University, Damascus, Syria; ${ }^{2}$ Department of Histo- \\ pathology, College of Medicine, Damascus University, Damascus, Syria. \\ Email: ${ }^{*}$ asfour_wasfi@yahoo.com
}

Received September $17^{\text {th }}, 2012$; revised October $24^{\text {th }}, 2012$; accepted November $15^{\text {th }}, 2012$

\begin{abstract}
A myriad of medicinal effects has been attributed to Thymoquinone (TQ), the major biological-active component of Nigella sativa. TQ has been shown to exhibit potent anti-tumor activities. The present work was undertaken to further explore TQ's chemopreventive efficacy against 1,2-dimethylhydrazine (DMH)-induced colon carcinogenesis in the rat model through a two-phase study (initiation and post-initiation) and to evaluate its potential impact on tumor progression and invasion in vivo. TQ treatment in the initiation phase significantly reduced tumor incidence, multiplicity and mean tumor volume. However, although mean tumor volume and multiplicity were decreased upon TQ treatment in the post-initiation phase, TQ did not reduce incidence significantly. Cellular proliferation, as assessed by expression of colonic PCNA, was shown to be inhibited in consequence to TQ treatment in both phases, with a more pronounced reduction in the initiation phase. In addition, our results demonstrated an appreciable negative impact of TQ on vascular endothelial growth factor (VEGF) production in tumor-bearing rats. Furthermore, we provided evidence that TQtreatment, in both phases, tended to considerably suppress tumor progression and invasion. Taken together, the present study demonstrated that TQ, at an orally daily dose of $10 \mathrm{mg} / \mathrm{kg}$, has a chemopreventive effect in the initiation phase, and has the potential to attenuate tumor burden, suppress progression of pre-neoplastic lesions and to inhibit tumor growth in the post-initiation phase of DMH-induced colon carcinogenesis, We surmise that such effects of TQ may be due to suppression of cellular proliferation and inhibition of VEGF production. The results could provide an effective chemopreventive approach in the primary prevention of colon cancer in humans in the next future, and illuminate a promising horizon to combat progression of benign colonic pre-neoplastic lesions into malignant metastatic tumors and to manage colon cancer.
\end{abstract}

Keywords: Thymoquinone; PCNA-LI; VEGF Serum Levels; Tumor Progression; Invasion; Rat; DMH-Induced Colon Carcinogenesis; Initiation; Post Initiation Phase; Modified Duke's Classification; Chemoprevention

\section{Introduction}

Cancer is the second leading cause of death in western countries surpassed only by cardiovascular disease [1].

According to the International Agency for Research on Cancer (IARC), it is estimated that by the year 2020 the global cancer burden will reach 16.8 million cases. These data suggest that cancer prevalence is on the rise and that it is rapidly becoming a global pandemic.

Colorectal cancer is the third most common malignant neoplasm worldwide [2]. In Syria, the incidence and mortality rates of colorectal cancer are increasing at an

${ }^{*}$ Corresponding author. alarming rate in recent decade, being the second most prevalent type of cancer (second to lung \& bronchus cancer in men, and breast cancer in women). It accounts for $12 \%$ of cancer incidence in males and females [3].

Colorectal carcinogenesis is a complex multi-step process that includes changes in both histomorphological appearances of the colonic mucosa and at molecular level. It is believed that there are two pathogenetically distinct pathways for the development of colon cancer $[4,5]$.

The first pathway is characterized by chromosomal instability resulting in stepwise accumulation of mutations in a series of oncogenes and tumor suppressor genes. The molecular evolution of colon cancer in this pathway can 
be seen through a series of morphologically identifiable stages: initial localized colonic epithelial hyperplasia, followed by formation of adenomas that progressively enlarge and ultimately develop into invasive cancers i.e. the adenoma-carcinoma sequence. The second pathway is characterized by genetic lesions in DNA mismatch repair genes (microsatellite instability). All the above mentioned series of genetic alterations destroy normal mechanisms controlling cellular growth, leading to abnormal cellular proliferation which is one of crucial mechanisms in carcinogenesis [6].

Most colon carcinomas, besides their genetic familial background, develop as a result of multiple assaults by various exogenous/endogenous carcinogenic compounds. It is well established that colon cancer may be ameliorated when risk factors such as life-style and environmental factors are modified [7].

Although complete removal of causative agents of cancer may not always be possible, chemoprevention of cancer using antimutagens and anticarcinogens present in foods $[8,9]$ or natural products (herbs and spices) has been suggested by various studies and much effort has been dedicated to a search for natural preventive agents, which would block or attenuate colorectal carcinogenesis process [10-12].

Epidemiological and experimental studies unveiled therapeutic potential of many culinary herbs and demonstrated the importance of compounds derived from plants in reducing the risk of colon cancer [13] and inhibiting the development and the multiplicity of tumors in experimental animal models [14]. Nigella sativa L. is an annual herbaceous plant belonging to the Ranunculaceae family, commonly used traditionally in Middle Eastern folk medicine as a natural remedy for various ailments for over 2000 years [15]. It is also used as a food additive and flavor in many countries. Thymoquinone (TQ), which is 2-Isopropyl-5-methyl-1,4-benzoquinone, is the abundant Nigella sativa essential oil compound and known to be the active principle responsible for many of the seed's antioxidant and anti-inflammatory effects [16].

TQ has been revealed to have promising anti-tumor effects. It inhibited the incidence and multiplicity of benzo (a) pyrene-induced fore-stomach tumors as well as 20-methylcholanthrene-induced fibrosarcoma tumors [17, 18]. The study of Badary [19] demonstrated that TQ attenuates ifosfamide-induced Fanconi syndrome in rats, enhances its anti-tumor activity in mice and increases the anti-tumor effects of ifosfamide. Furthermore, TQ was shown to reduce cisplatin-induced nephrotoxicity without disturbing its anti-tumor activity [20].

In vitro studies reported the ability of TQ to inhibit the proliferation of colon cancer cells [21-23], lymphoblastic leukemia cells [24], laryngeal carcinoma cells $[25,26]$, pancreatic cells [27], and prostate cancer cells [26-28] and so on.

All of these promising results and encouraging findings make TQ an interesting compound that merits further evaluation.

The purpose of the following research is to appraise its chemopreventive effects (in vivo) in the rat model of DMH-induced colon cancer and to evaluate its roles in suppressing cellular proliferation, inhibiting tumor growth and in obstructing tumor progression and invasion in this animal model through a two-phase-study: an initiation phase and a post-initiation phase.

\section{Materials and Methods}

\subsection{Materials}

\section{Animals and Chemicals}

Five-week-old male albino wistar rats were purchased from LEEN Company for laboratory animals (DamascusSyria).

Upon arrival to the animal care facility (Faculty of Pharmacy-Damascus university), rats were housed four per polypropylene cage with a wire-mesh top and a hygienic bed of sawdust in a specific pathogen-free animal room under controlled conditions (at $24^{\circ} \mathrm{C} \pm 1{ }^{\circ} \mathrm{C}, 50 \% \pm$ $10 \%$ relative humidity with an altering $12 \mathrm{~h}$ light $/ 12 \mathrm{~h}$ dark cycle, and 20 air changes/hour) and had unrestricted access to a basal commercial rat pellet diet and tap water ad libitum.

All the procedures were performed in accordance with the standard guidelines for care and use of laboratory animals and the protocols followed were approved by the local University Ethics Committee for Animal Research.

1,2-Dimethylhydrazine dihydrochloride (DMH) was purchased from Acros Organics (Morris Plains, NJ, USA) and Thymoquinone (TQ) was obtained from Sigma-Aldrich (St. Louis, MO, USA).

\subsection{Methods}

\subsubsection{Preparation of Carcinogen}

1, 2-Dimethylhydrazine dihydrochloride (DMH) was dissolved in $1 \mathrm{mMol} / \mathrm{L}$ EDTA-normal saline; the $\mathrm{pH}$ was adjusted to 6.5 with $1 \mathrm{Mol} / \mathrm{L} \mathrm{NaOH}$ to ensure the $\mathrm{pH}$ suitability and the stability of the chemical. The resultant solution of the carcinogen was used immediately after preparation.

A full-face respirator equipped with a combination filter cartridge (The Advantage ${ }^{\circledR}$ 1000: MSA Co., USA) was used during the preparation of the carcinogen solution and when injecting animals.

DMH was injected subcutaneously (s.c.) at a dose of $40 \mathrm{mg} / \mathrm{kg} /$ body weight once a week for ten consecutive weeks on the dorsal back of the animal. 


\subsubsection{Experimental Design}

After one week of acclimatization the eighty rats were randomly assigned into five groups.

Animals in Group I: $(n=16)$ served as negative controls. Rats in this group were also injected subcutaneously, at weekly intervals for 10 weeks, with $0.5 \mathrm{ml}$ of 1 $\mathrm{mMol} / \mathrm{L}$ EDTA-saline which was used as a vehicle for the (DMH) treatments (Normal control group).

Animals in Group II: $(n=16+8)$ served as carcinogen controls or positive controls. They were given subcutaneous injections of (DMH) once a week at a dose of 40 $\mathrm{mg} / \mathrm{kg}$ body weight for 10 consecutive weeks (DMH group).

8 animals of this group were sacrificed at the end of the initiation phase (i.e. in the eleventh week) to identify the histological alterations in the colonic histoarchitecture at this stage of the carcinogenesis process.

Group III animals $(n=16)$ received the same DMH treatment as in group II and simultaneously gavaged thymoquinone at a daily dose of $10 \mathrm{mg} / \mathrm{kg}$ body weight for the whole 10 weeks period of the initiation phase (DMH + TQ initiation group).

Animals in Group IV $(n=16)$ were subjected to the same carcinogen protocol as in group II and subsequently gavaged thymoquinone at a daily dose of $10 \mathrm{mg} / \mathrm{kg} /$ body weight during the last 11 post-initiation weeks of the experimental period (DMH + TQ post-initiation group)

And Animals in Group V $(n=8)$ were intubated thymoquinone alone at a daily dose of $(10 \mathrm{mg} / \mathrm{kg}$ body weight) for the whole period of the experiment (22 weeks) (TQ group).

Table 1 summarizes the design of the experiment.

\subsubsection{Record of Body Weights}

A record of the body weights of normal control, DMH and TQ-treated animals was kept throughout the study. The animals were weighed at the beginning of the experiment, once a week during the experiment and finally before sacrifice.

\subsubsection{Blood Samples}

By the end of week 22, blood samples were collected by hurt puncture under ether anesthesia. Blood samples were allowed to clot for 2 hours at room temperature before centrifuging for 20 minutes at $1000 \times \mathrm{g}$ (according to the instructions of the ELISA kit). Serums were removed, aliquoted and stored at $-80^{\circ} \mathrm{C}$ until they were assayed.

Serum levels of VEGF were determined using enzyme-linked immunosorbent assay (ELISA) kit (Quantikine ${ }^{\circledR}$ Rat VEGF ELISA Kit, R\&D systems Inc., Minneapolis, MN, USA).

\subsubsection{Tissue Processing}

After blood samples were withdrawn, Rats were sacrificed (by an excessive dose of anesthetic ether). They were then autopsied, and the colons were excised, opened along the longitudinal median axis, flushed with saline, and the inner surface was examined macroscopically. The number of tumors in each colon was counted. The length (L), width (W) and height $(\mathrm{H})$ of tumor were measured using a vernier caliper with $0.1 \mathrm{~mm}$ graduations, and Tumor Volume (TV) was calculated $(\mathrm{TV}=\mathrm{L} \times$ $\mathrm{W} \times \mathrm{H} \times \pi / 6$ ).

The chemopreventive tumor response was assessed on the basis of tumor incidence, multiplicity and tumor volume which were calculated as follows: Tumor incidence, percentage of animals having tumors; Tumor multiplicity, mean of tumors counted/animals. Mean tumor volume (mean volume of tumors in tumor-bearing rats.

After the gross pathologic changes (number, dimensions and distribution of tumors) were recorded, the colons were fixed flat between pieces of filter paper in 10 $\%$ phosphate-buffered formalin for $24 \mathrm{~h}$.

Afterwards, tissue samples were processed for paraffin embedding, cut into $5-\mu \mathrm{m}$ thick sections and stained with routine hematoxylin and eosin $(\mathrm{H} \& \mathrm{E})$ for histological diagnosis of tumors. Other paraffin 5- $\mu \mathrm{m}$ thick sections were employed for immunohistochemical staining.

The liver, kidneys and other major organs (stomach, small intestine, spleen, lungs and lymph nodes) were also excised, fixed in $10 \%$ phosphate-buffered formalin and then prepared for histological analysis.

Colons of the eight rats from DMH group, sacrificed at the end of the 10th week, were prepared at the time and stained with (H\&E) for histopathological observations at the light microscopic level.

Table 1. Exprimental design.

\begin{tabular}{|c|c|c|}
\hline Experimental Groups & Number of rats & Protocol \\
\hline Normal control & $(n=16)$ & (s.c) injections of mMol/L EDTA-saline (vehicle of DMH) for 10 consecutive wk. \\
\hline DMH & $(n=16+8)$ & $\begin{array}{l}\text { Weekly (s.c) injections of (DMH) at a dose of }(40 \mathrm{mg} / \mathrm{kg}) \text { for } 10 \text { consecutive wk. } \\
\text { (Eight rats were sacrificed at the end of the } 10 \text { th wk). }\end{array}$ \\
\hline DMH + TQ initiation & $(n=16)$ & Same DMH treatment + simultaneously TQ-treated $(10 \mathrm{mg} / \mathrm{kg} /$ day $)$ for $10 \mathrm{wk}$. \\
\hline DMH + TQ post-initiation & $(n=16)$ & Same DMH treatment, subsequently TQ-treated $(10 \mathrm{mg} / \mathrm{kg} /$ day $)$ from the 11 th to the $22 \mathrm{nd}$ wk. \\
\hline TQ & $(n=8)$ & Daily dose of TQ $(10 \mathrm{mg} / \mathrm{kg})$ for $22 \mathrm{wk}$ \\
\hline
\end{tabular}




\subsubsection{Immunohistochemical (IHC) Analysis of PCNA}

Briefly, paraffin sections were deparaffinized in xylene, rehydrated through graded ethanol solutions to distilled water, and washed in PBS.

PCNA immunohistochemical staining was performed following the instructions of the employed Peroxidase/DAB detection kit (EnVision ${ }^{\mathrm{TM}+} / \mathrm{HRP}, \mathrm{DAKO}$, Glostrup, Denmark). Monoclonal mouse anti-PCNA (clone PC10, 1:200 dilution; Dako) antibodies were used. Sections were then counter-stained for 1 - 2 min with hematoxylin (Sigma, St. Louis, MO, USA). Sections were observed under a light microscope and The PCNA labeling index (LI) was determined.

\subsubsection{Statistical Analysis}

All Statistical analyses were performed using The (GraphPad Prism5) statistical software. The significance of differences in lesion (tumor) incidences between the groups was assessed by Fisher's exact test. Differences in mean body weight, Multiplicity, tumor volume, PCNA labeling index and VEGF serum levels in the different groups were compared by One-way Analysis of Variance (ANOVA) followed by Tukey's Multiple Comparison Test. All tests were performed two-sided. Statistical significance, concerning differences in histological grades of colon tumors among the groups, was determined by KruskalWallis test followed by Dunn's Multiple Comparison test. Correlations were analyzed by performing Spearman correlation coefficient analysis for independent variables. The statistical significance was set at $(P<0.05)$.

\section{Results}

\subsection{General Observation}

All rats survived to the final termination and maintained a relatively healthy appearance throughout the experiment. No apparent signs of severe toxicity were observed in animals received TQ.

By the end of 22nd week, final average body weight of the rats in $(\mathrm{DMH})$ group was significantly decreased compared with the normal control group $(P<0.05)$. Body weights of the rats in both (DMH + TQ initiation) group and (DMH + TQ post-initiation) group were decreased; however, the reductions were not statistically significant when compared to the normal control group. On the contrary, when compared with the DMH group, average body weight of both TQ-treated groups was higher (TQ treatment tended to improve body weight gain in the initiation and post-initiation phases).

Results of average body weights are shown in Table 2.

Histopathological analysis of colons of rats $(n=8)$ from DMH group, sacrificed at the end of the 10th week (end of the initiation phase), revealed colonic hyperplasia at that point of the experiment, which was the starting point for the post-initiation phase.

\subsection{Tumor Incidence}

Table 3 summarizes the incidences of DMH-induced colon tumors in the DMH group and TQ-treated groups. Macroscopically, the distribution of colon tumors was

Table 2. Average body weights.

\begin{tabular}{cccc}
\hline Group & Number of Rats & \multicolumn{2}{c}{ Body weight (g) } \\
\cline { 2 - 4 } I Normal (Negative) control & & Day 0 & $410.22 \pm 19.1$ \\
II DMH (Carcinogen control) & 16 & $76.12 \pm 7.81$ & $334.17 \pm 23.5^{\mathbf{a}}$ \\
III DMH + TQ initiation Phase & 16 & $78.69 \pm 8.11$ & $79.42 \pm 6.99$ \\
IV DMH + TQ Post initiation Phase & 16 & $77.63 \pm 9.11$ & $399.54 \pm 17.4^{\mathbf{b}}$ \\
V TQ & 8 & $74.98 \pm 6.51$ & $407.44 \pm 15.6$ \\
\hline
\end{tabular}

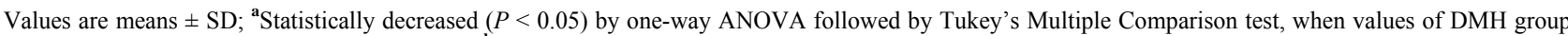
were compared with the normal control group; 'significantly higher $(P<0.05)$ by one-way ANOVA followed by Tukey's Multiple Comparison test, when values were compared with DMH group.

Table 3. Chemopreventive efficacy of orally administered Thymoquinone $(10 \mathrm{mg} / \mathrm{kg} / \mathrm{day})$ on tumor incidence, tumor multiplicity and tumor volume of DMH-induced rat colonic tumors.

\begin{tabular}{cccc}
\hline Group & Incidence No. (\%) & Multiplicity & Tumor volume (mm ${ }^{3}$ ) \\
\hline Normal control & $0(0)$ & 0 & - \\
DMH & $16(100)$ & $5.3 \pm 2.19$ & $518.1 \pm 61.13$ \\
DMH + TQ initiation & $11(68.75)^{\mathrm{a}}$ & $1.93 \pm 1.15^{\mathrm{b}, \mathrm{c}}$ & $175.88 \pm 42.35^{\mathrm{d}, \mathrm{e}}$ \\
DMH + TQ Post initiation & $13(81.25)$ & $3.57 \pm 1.64^{\mathrm{b}}$ & $301.44 \pm 56.27^{\mathrm{d}}$ \\
TQ (alone) & $0(0)$ & 0 & - \\
\hline
\end{tabular}

Data are expressed as mean \pm S.D. 'Significantly lower in comparison with DMH group using Fisher's exact test, $P<0.05$; ${ }^{\mathrm{b}}$ Significantly lower in comparison with DMH group using one-way-ANOVA followed by Tukey's Multiple Comparison test, $P<0.05$; ${ }^{\mathrm{c}}$ Significantly lower when compared with (DMH + TQ

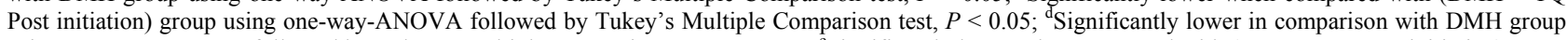

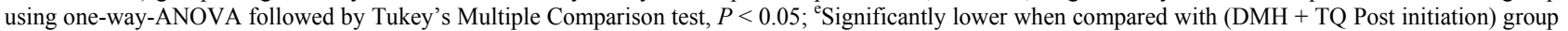
using one-way-ANOVA followed by Tukey's Multiple Comparison test, $P<0.05$. 
predominantly observed in the distal and middle colon at the end of 22nd week. All rats in DMH group developed colon cancer at the end of this study whereas, neither the animals of normal control group nor those of TQ (alone) group developed colon tumors.

Note:

Two rats in (DMH) group developed an ear tumor each. Histopathalogical analysis of the tumors revealed Squamous Papilloma.

\subsection{Tumor Multiplicity}

In addition to tumor incidence, there were significant differences in tumor multiplicity, or number of tumors per rat, among the different groups. Data are shown in Table 3.

\subsection{Tumor Volume}

Mean tumor volume was significantly different among the treatment groups. Table $\mathbf{3}$ explains these differences.

\subsection{Tumors Grading}

Animals with DMH-induced colon cancer characteristically developed multiple tumors and each tumor was at a different histological stage. Consequently, for the purpose of this experiment, animals were staged with reference to a single index tumor, defined as the largest macroscopically and histologically identifiable colon tumor.

Colon tumors were divided into adenoma and carcinoma. Grades of carcinoma were allocated according to modified Duke's pathological classification [29].

Four cases of mucinous adenocarcinoma were observed in the positive control (DMH) group, and two cases in (DMH + TQ post-initiation) group. Although mucinous adenocarcinoma is considered a more aggressive subtype of adenocarcinoma associated with particularly poor prognosis, all mucinous adenocarcinoma cases in the present study were classified as carcinoma and graded according to modified Duke's staging.

Histopathological findings are summarized in Table 4.

\subsection{Serum Levels of Vascular Endothelial Growth Factor}

Significant differences in serum concentrations of VEGF were detected among the various groups.

Figure 1 clarifies these differences.

\subsection{Immunohistochemical Analysis of PCNA}

Figure 2 shows the results of immunohistochemical analysis of PCNA-LI. After 22 weeks, the PCNA-LI in (DMH) group increased significantly over the normal control group. Oral administration of TQ (in both phases) diminished the PCNA-LI $(P<0.05)$.

\section{Discussion}

Extensive research over the past decade has shown that TQ exhibited inhibitory effects on cellular proliferation of many types of cancer cell lines, including breast adenocarcinoma, ovarian adenocarcinoma [30], colorectal cancer [31], human pancreatic adenocarcinoma, uterine sarcoma [32], neoplastic keratinocytes [33], human osteosarcoma [34], fibrosarcoma, lung carcinoma [35] and hormone-refractory prostate cancer [35] and so on.

Although TQ anticarcinogenic potential has been linked with an impressive amount of data primarily from human cell culture systems, emerging results of cancer prevention in laboratory animal models provide evidence that TQ has an anticancer activity not only in vitro but also in vivo [35-37].

The present (in vivo) study, performed on the rat model of DMH-induced colon cancer, has demonstrated that administration of TQ at an orally daily dose of (10 $\mathrm{mg} / \mathrm{kg}$ body weight) during the initiation phase exhibited a remarkable chemopreventive effect against colon carcinogenesis. We have demonstrated that TQ prevented tumorigenesis (incidence) to a considerable extent, attenuated tumor burden (multiplicity) and, concomitantly, inhibited tumor growth. Tumor incidence was markedly reduced by about $31 \%$, while the mean number of tumors per rat (i.e. Multiplicity) decreased by nearly $64 \%$. Thy-

Table 4. Numbers of rats with DMH-induced colon tumors allocated to one of six histological grades.

\begin{tabular}{ccccccc}
\hline \multirow{2}{*}{ Group } & Adenoma $n$ & \multicolumn{4}{c}{ Carcinoma } \\
\cline { 3 - 7 } & & Duke's A $\boldsymbol{n}$ & Duke's B1 $\boldsymbol{n}$ & Duke's B2 $\boldsymbol{n}$ & Duke's C1 $\boldsymbol{n}$ & Duke's C2 $\boldsymbol{n}$ \\
\hline DMH & 0 & 0 & 0 & 11 & 3 & 2 \\
DMH + TQ (I.) & $\mathbf{a}$ & 3 & 3 & 1 & 0 & 0 \\
DMH + TQ (P.I.) & 2 & 3 & 3 & 3 & 2 & 0 \\
\hline
\end{tabular}

$n$ : Number of Rats in each histological grade. ${ }^{\mathrm{a}}$ Histological grade of (DMH+TQ initiation) group is less progressive and less invasive when compared with DMH (carcinogen control) group. Statistical significance was assessed by Kruskal-Wallis test followed by Dunn's Multiple Comparison test; ${ }^{\mathbf{b}}$ Less progressive (and less invasive) in comparison with DMH (carcinogen control) group by Kruskal-Wallis test followed by Dunn's Multiple Comparison test, $(P<0.05)$. 


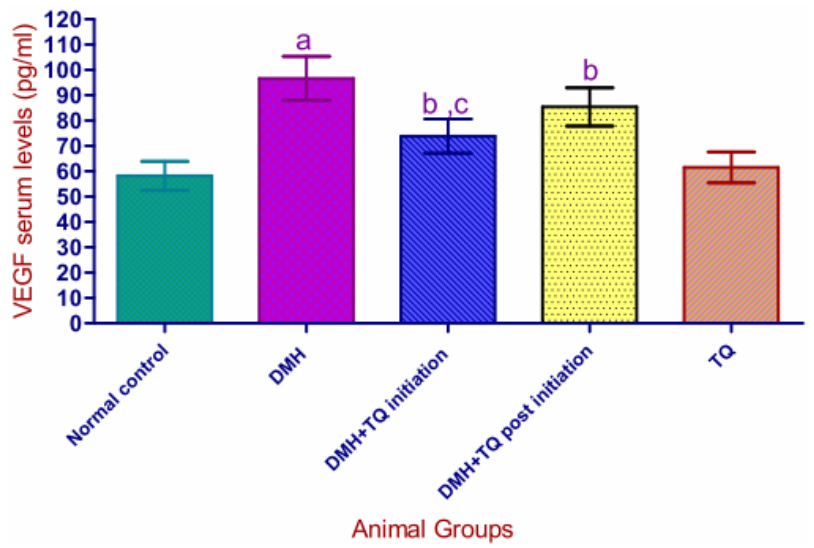

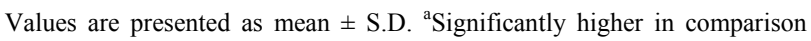
with normal control group using one-way-ANOVA followed by Tukey's Multiple Comparison test, $P<0.05$; ${ }^{\text {b }}$ Significantly lower when compared to DMH group using one-way-ANOVA followed by Tukey's Multiple Comparison test, $P<0.05$; 'Significantly lower when compared with (DMH + TQ Post initiation) group using one-way-ANOVA followed by Tukey's Multiple Comparison test, $P<0.05$; Serum levels of VEGF were significantly correlated with tumor volume by Spearman's coefficient analysis $P<$ 0.05 .

Figure 1. Serum levels of VEGF in the experimental groups.

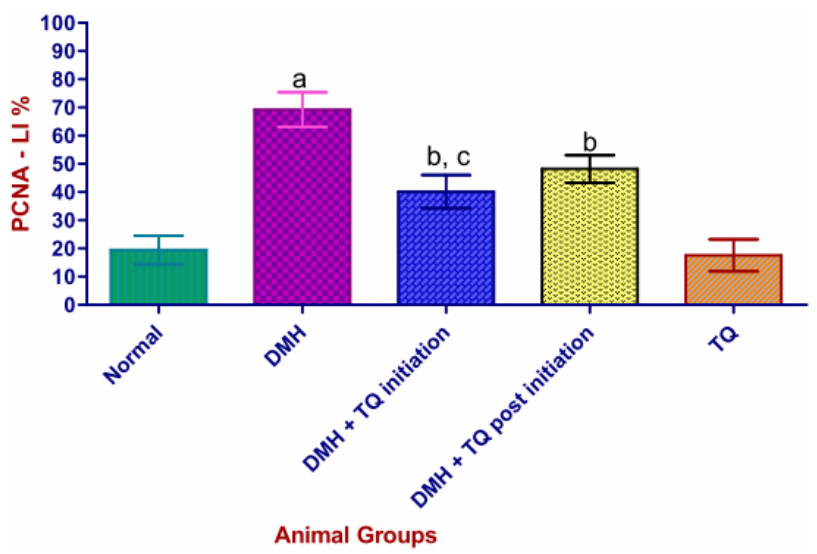

PCNA-LI: proliferating cell nuclear antigen-Labeling Index. Values are

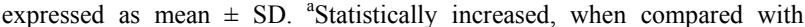
normal control group using one-way-ANOVA followed by Tukey's Multiple Comparison test, $(P<0.05)$; ' Statistically decreased, when compared with DMH group using one-way-ANOVA followed by Tukey's Multiple Comparison test, $(P<0.05)$; 'Statistically lower than (DMH + TQ Postinitiation) group $(P<0.05)$ by one-way-ANOVA followed by Tukey's Multiple Comparison test.

Figure 2. Effect of Thymoquinone on PCNA-LI in colonic mucosa of DMH-treated rats.

moquinone treatment in the initiation phase diminished tumor volume dramatically by $66 \%$.

In a mouse model of DMH-induced colon cancer, Gali et al. [16] reported that TQ injected intraperitoneally (i.p.) significantly reduced the numbers and sizes of aberrant crypt foci (ACF) by $86 \%$ and reduced tumor multiplicity at week 20 from 17.8 in the $\mathrm{DMH}$ group to 4.2 in mice injected with TQ, implicating a reduction of $76.4 \%$. It was also reported that, in a xenograft model of HCT116 colon cancer cells, TQ significantly delayed the growth of the tumor cells.

In addition, administration of TQ (10 mg/kg/i.p.) for 18 days has been shown to inhibit Lung (LNM35 cells) tumor growth by $39 \%$ in mice [38].

Tingfang et al. [39] concluded that TQ effectively inhibited prostate tumor growth (in xenograft mouse model) at small tumor stage $\left(50 \mathrm{~mm}^{3}\right)$ at the dose of $6 \mathrm{mg} / \mathrm{kg} /$ day for 15 days.

On the other hand, although the present study showed that post-initiation treatment with TQ significantly reduced multiplicity by about $33 \%$ and inhibited tumor growth by $42 \%$, TQ treatment in the post-initiation phase did not reduced the incidence of colon tumors significantly.

The insignificant impact of TQ on tumor incidence in the post-initiation phase may be attributed to the fact that the initiation protocol of DMH was too strong in our study (40 mg/kg/week for ten consecutive weeks) which made TQ, at the employed dose level, unable to completely hinder the tumorigenesis process and to adequately suppress cellular proliferation (at this stage of histological changes) and to abolish or totally prevent tumor formation (tumor appearance), which led to more pronounced tumor incidence within animals in the postinitiation phase. In other words TQ possibly targets more effectively the earliest stages in colon carcinogenesis.

More appropriate TQ dosing in the post-initiation phase and the possible ability of TQ to inhibit enzymes responsible for DMH metabolism (i.e. reducing active metabolites of the carcinogen) in the initiation phase should be considered and further evaluated to elucidate the difference in TQ effects on tumor incidence between the initiation phase and the post-initiation phase.

In addition to the aforementioned significant reductions in mean tumor volume and multiplicity in animals received TQ in both phases (initiation and the post-initiation) of the tumorigenesis process, immunohistochemical staining revealed a concomitant significant decrease in the colonic PCNA-LI of the TQ-treated groups when compared with the carcinogen control (DMH) group. This decrease was more pronounced in the initiation phase than in the post-initiation phase.

It is well known that PCNA is an auxiliary protein of the DNA polymerase delta, reaching an expression peak during the S-phase of the cell cycle and playing an important role in cellular proliferation $[40,41]$.

PCNA-LI has been used as an intermediate biomarker in chemoprevention of colorectal cancer [42]. It was observed [43] that vitamin A significantly decreased PCNA$\mathrm{LI}$ in the AOM-induced colorectal cancer animal model. Another study gave similar result [44].

Jia X.D. and Han C. [45] indicated that the chemopre- 
ventive effect of both green tea and tea pigments against DMH-induced colorectal cancer in wistar rats was associated with a significant inhibition of colonic PCNA-LI. It was concluded that suppression of cellular proliferation may represent one of the mechanisms through which green tea and tea pigments exert their chemopreventive effects.

In addition, it is well established that increased PCNALI in colonic aberrant crypt foci (ACF), the known preneoplastic lesions, leads to higher risk for malignant progression $[46,47]$.

Furthermore, tumors with higher growth fractions, as assessed by expression of PCNA, behave more aggressively than tumors with lower growth fractions [48].

Accordingly, and in view of our experimental findings regarding PCNA immunohistochemical staining and the statistically significant decreases in colonic PCNA-LI in TQ-treated groups, our results implicated that TQ exhibited a chemopreventive effect against DMH-induced colon cancer in the initiation phase and that TQ had the potential to inhibit/slow colon tumorigenesis and to obstruct the growth and progression of hyperplastic foci into frank invasive tumors in the post-initiation phase in this rat model. These effects of TQ are mediated, at least in part, through its ability to suppress cellular proliferation.

In vitro studies demonstrated that TQ inhibits the proliferation of cancer cells and induces apoptotic cell death [21-24] via p53-dependent [31] and p53-independent [23] \& [34] pathways; another report showed that, in the p53 mutant leukemic Jurkat cells, TQ induces apoptotic cell death via a p73-dependent pathway [24].

Whether TQ induces apoptosis in our animal model of DMH-induced colon cancer and the possible mechanisms underlying this apoptotic efficacy are being investigated in our laboratory and the primary results are encouraging.

The Duke's staging system for human colorectal cancer provides accurate prognostic information. In other words, cases with less advanced tumors (stage A) can survive significantly longer than those with more advanced tumors (stage B and C), irrespective of treatment. In the present study, the histopathological analysis of tumors and the histological grading of carcinoma (using modified Duke's classification) indicated that rats in TQtreated groups tended to develop considerably less progressive tumors and that the extent to which carcinomas invade through the histological layers of the colon was remarkably reduced.

In vitro studies provided evidence that TQ effectively inhibited human umbilical vein endothelial cell (HUVEC) migration and invasion [39].

In addition, it was reported that TQ at non-toxic concentrations inhibited the invasive potential of lung
(LNM35), breast (MDA-MB-231) cancer cells [38]. Our (in vivo) study demonstrated, for the first time in the rat model of DMH-induced colon cancer, that TQ treatment in both phases (initiation and post-initiation) effectively inhibited progression and invasion. Two animals of DMH group developed carcinomas classified as Duke's stage C2 implicating increased invasion (tumor penetrates through muscularis propria), lymph node involvement and poorer prognosis, while none of the animals of TQtreated groups had such an advanced stage, suggesting an improved prognosis and less progression.

It is worth mentioning that the differences encountered among the experimental groups concerning the incidence of mucinous adenocarcinoma must be taken into consideration when evaluating the protective effect of TQ against induced colon cancer in vivo.

The overall histological findings clearly indicate and expand the database that TQ might play promising anticancer role with respect to colon carcinogenesis. Moreover, the absence of tumor incidence in rats treated with TQ alone for a long period ( $22 \mathrm{wk}$ ) suggests TQ, at this dose level, causes no disruption of normal cellular homeostasis and hence is non-toxic.

The progression and growth of most solid tumors and their subsequent metastases depends critically on angiogenesis because, without the development of new blood vessels, tumors cannot grow beyond 2 - $3 \mathrm{~mm}$ [49].

In addition, neovascularization increases the possibility that tumor cells will enter the circulation and gives rise to metastases [50].

The accumulated evidence has confirmed the importance of angiogenesis and validated the theory that inhibition of neovascularization is an attractive target and a promising anti-cancer strategy [51-54]. Bevacizumab is the fruit of intensive efforts in this domain $[54,55]$.

The process of angiogenesis is tightly controlled by both stimulatory and inhibitory factors [56,57]. Vascular endothelial growth factor (VEGF) is the pre-eminent, most potent member of a large family of angiogenic cytokines that have been identified [56]. VEGF is believed to have a leading position in the hierarchy of mediators of both normal and tumoral angiogenesis [56].

Indeed, many tumor cell lines secrete VEGF in vitro, and high VEGF mRNA levels can be detected by in situ hybridization in most human tumors, including gastrointestinal carcinoma [58].

Both VEGF and its receptor are expressed at high levels in metastatic human colon carcinomas [59] and high preoperative VEGF concentrations were found to be associated with reduced overall survival [60-62].

Several studies reported that serum VEGF is an independent prognostic factor [63-66]. It has been shown [67] that increased preoperative circulating VEGF levels cor- 
relate with factors reflecting disease progression including tumor size, and nodal and distant metastases in colorectal cancer.

In our animal model of DMH-induced colon cancer, the results indicated that serum levels of VEGF in all animals that developed tumors were significantly elevated in comparison with the normal control animals.

It was remarkable that VEGF production is increased with advancing Duke's stages (which reflect disease progression) and that circulating VEGF levels were correlated with tumor volume.

Thus, we surmise that serum VEGF might be an easy to determine biomarker or indicator for tumor growth and for tumor progression which might be beneficial for monitoring tumoral status in animal studies concerning colon cancer therapy. However, further studies are warranted and more evaluation and validation need to be dedicated.

Our results also showed that serum levels of VEGF in the TQ-treated groups were significantly lower than those measured in the positive control group (DMH), suggesting that TQ had the potential to inhibit VEGF production and thereby can exert a negative impact on tumoral angiogenesis, Consequently, It could be that suppressive effect of TQ on VEGF production which contributes to the chemopreventive and tumor growth inhibitory effects (in vivo) in this animal model of colon carcinogenesis.

A previous study [39] reported that TQ prevented human prostate tumor growth in xenograft mouse models through inhibiting tumor-angiogenesis. The study showed that TQ inhibited angiogenesis by suppressing the activation of VEGF-induced ERK and AKT.

It is established that AKT signaling stimulates the production of hypoxia inducible factor $\alpha(\mathrm{HIF} \alpha)$ transcription factors, thereby mediates secretion of VEGF and other growth factors which are important pro-angiogenic factors $[68,69]$. Thus, a resultant effect of suppressing the activation of AKT signaling is inhibited VEGF production, and consequently, inhibited angiogenesis or neovascularization.

Erdurmus M. et al. [70] reported that TQ showed inhibitory effects on corneal neovascularization in the rat model.

\section{Conclusion}

In conclusion, the present study demonstrated that TQ, the major biological-active component of the natural medicine Nigella sativa, at an orally daily dose of (10 $\mathrm{mg} / \mathrm{kg}$ body weight) has a chemopreventive effect against DMH-induced colon carcinogenesis in the rat model during the initiation phase. This could provide an effective chemopreventive approach in the primary prevention of colon cancer in humans in the next future. Our study also provided evidence that TQ appreciably attenuated tumor burden and suppressed the progression of preneoplastic lesions and inhibited tumor growth and invasion in the post-initiation phase, which might make TQ a promising agent to combat the progression of benign polyps and other pre-neoplastic lesions into malignant metastatic tumors and to manage colon cancer. We content that TQ potential to suppress cellular proliferation and to inhibit VEGF production might be important means through which TQ inhibits chemically induced colon tumorigenesis.

\section{REFERENCES}

[1] J. Ferlay, F. Bray, P. Pisani and D. Parkin, "GLOBOCAN 2002: Cancer Incidence, Mortality and Prevalence Worldwide," IARC Cancer Base, Vol. 5, IARC Press, Lyon, 2004.

[2] J. Ferlay, H. R. Shin, F. Bray, D. Forman, C. Mathers and D. M. Parkin, "GLOBOCAN 2008 v1.2, Cancer Incidence and Mortality Worldwide," IARC Cancer Base, Vol. 2, International Agency for Research on Cancer Press, Lyon, 2010. http://globocan.iarc.fr

[3] Z. Msallati, A. El Jord and F. Al-Jerf, "Cancer Incidence in Syria, National," Cancer Registry, Cancer Prevention Directorate, Ministry of Health, Damascus, 2009.

[4] J. R. Jass, "Pathogenesis of Colorectal Cancer," Surgical Clinics of North America, Vol. 82, No. 5, 2002, pp. 891904. doi:10.1016/S0039-6109(02)00047-6

[5] V. Kumar, A. K. Abbas and N. Fausto, "Robbins and Cotran Pathologic Basis of Diseases," 7th Edition, Saunders, Philadelphia, 2004.

[6] I. Weinstein, "The Origin of Human Cancer: Molecular Mechanisms and Their Implications for Cancer Prevention and Treatment," Cancer Research, Vol. 48, No. 15 1988, pp. 4135-4143.

[7] W. C. Willett, "Balancing Life-Style and Genomics Research for Disease Prevention," Science, Vol. 296, No. 5568, 2002, pp. 695-698. doi:10.1126/science.1071055

[8] Y. S. Kim and J. A. Milner, "Dietary Modulation of Colon Cancer Risk," Journal of Nutrition, Vol. 137, No. 11, 2007, pp. 2576-2579.

[9] A. E. Tammariello and J. A. Milner, "Mouse Models for Unraveling the Importance of Diet in Colon Cancer Prevention," Journal of Nutritional Biochemistry, Vol. 21, No. 2, 2010, pp. 77-88. doi:10.1016/j.jnutbio.2009.09.014

[10] D. E. Corpet and F. Pierre, "How Good Are Rodent Models of Carcinogenesis in Predicting Efficacy in Humans? A Systematic Review and Meta-Analysis of Colon Chemoprevention in Rats, Mice and Men," European Journal of Cancer, Vol. 41, No. 13, 2005, pp. 1911-1922. doi:10.1016/i.ejca.2005.06.006

[11] M. J. Wargovich, A. Jimenez, K. McKee, et al., "Efficacy of Potential Chemopreventive Agents on Rat Colon Ab- 
errant Crypt Formation and Progression," Carcinogenesis, Vol. 21, No. 6, 2000, pp. 1149-1155. doi:10.1093/carcin/21.6.1149

[12] M. Perše and A. Cerar, "Morphological and Molecular Alterations in 1,2 Dimethylhydrazine and Azoxymethane Induced Colon Carcinogenesis in Rats," Journal of Biomedicine and Biotechnology, 2011, Article ID: 473964, $14 \mathrm{p}$

[13] M. Lipkin, B. Reddy, H. Newmark and S. A. Lamprecht, "Dietary Factors in Human Colorectal Cancer," Annual Review of Nutrition, Vol. 19, 1999, pp. 545-586. doi:10.1146/annurev.nutr.19.1.545

[14] Y. J. Surh, "Cancer Chemoprevention with Dietary Phytochemicals," Nature Reviews Cancer, Vol. 3, No. 10, 2003, pp. 768-780. doi:10.1038/nrc1189

[15] H. Jrah Harzallah, B. Kouidhi, et al., "Chemical Composition, Antimicrobial Potential against Cariogenic Bacteria and Cytotoxic Activity of Tunisian Nigella Sativa Essential Oil and Thymoquinone," Food Chemistry, Vol. 129, No. 4, 2011, pp. 1469-1474. doi:10.1016/j.foodchem.2011.05.117

[16] H. Gali-Muhtasib, M. Ocker, D. Kuester, et al., "Thymoquinone Reduces Mouse Colon Tumor Cell Invasion and Inhibits Tumor Growth in Murine Colon Cancer Models," Journal of Cellular and Molecular Medicine, Vol. 12, No. 1, 2008, pp. 330-342. doi:10.1111/j.1582-4934.2007.00095.x

[17] O. A. Badary and A. M. Gamal El-Din, "Inhibitory Effects of Thymoquinone against 20-MethylcholanthreneInduced Fibrosarcoma Tumorigenesis," Cancer Detection and Prevention, Vol. 25, No. 4, 2001, pp. 362-368.

[18] O. A. Badary, O. A. Al-Shabanah, M. N. Nagi, et al., "Inhibition of Benzo (a) Pyrene-Induced Forestomach Carcinogenesis in Mice by Thymoquinone," European Journal of Cancer Prevention, Vol. 8, No. 5, 1999, pp. 435-440. doi:10.1097/00008469-199910000-00009

[19] O. A. Badary, "Thymoquinone Attenuates IfosfamideInduced Fanconi syndrome in Rats and Enhances Its Antitumor Activity In mice," Journal of Ethnopharmacology, Vol. 67, No. 2, 1999, pp. 135-142. doi:10.1016/S0378-8741(98)00242-6

[20] O. A. Badary, M. N. Nagi, O. A. Al-Shabanah, et al., "Thymoquinone Ameliorates the Nephrotoxicity Induced by Cisplatin in Rodents and Potentiates Its Antitumor Activity," Canadian Journal of Physiology and Pharmacology, Vol. 75, No. 12, 1997, pp. 1356-1361. doi:10.1139/y97-169

[21] N. El-Najjar, M. Chatila, H. Moukadem, et al., "Reactive Oxygen Species Mediate Thymoquinone-Induced Apoptosis and Activate ERK and JNK Signaling," Apoptosis, Vol. 15, No. 2, 2010, pp. 183-195. doi:10.1007/s10495-009-0421-z

[22] H. Gali-Muhtasib, D. Kuester, C. Mawrin, et al., "Thymoquinone Triggers Inactivation of the Stress Response Pathway Sensor CHEK1 and Contributes to Apoptosis in Colorectal Cancer Cells," Cancer Research, Vol. 68, No. 14, 2008, pp. 5609-5618.

[23] M. A. El-Mahdy, Q. Zhu, Q. E. Wang, et al., "Thymo- quinone Induces Apoptosis through Activation of Caspase-8 and Mitochondrial Events in p53-Null Myeloblastic Leukemia HL-60 Cells," International Journal of Cancer, Vol. 117, No. 3, 2005, pp. 409-417. doi:10.1002/ijc. 21205

[24] M. Alhosin, A. Abusnina, M. Achour, et al., "Induction of Apoptosis by Thymoquinone in Lymphoblastic Leukemia Jurkat Cells Is Mediated by a p73-Dependent Pathway Which Targets the Epigenetic Integrator UHRF1," Biochemical Pharmacology, Vol. 79, No. 9, 2010, pp. 1251-1260.

[25] K. Womack, M. Anderson, M. Tucci, et al., "Evaluation of Bioflavonoids as Potential Chemotherapeutic Agents," Biomedical Sciences Instrumentation, Vol. 42, 2006, pp. 464-469.

[26] L. R. Richards, P. Jones, J. Hughes, et al., "The Physiological Effect of Conventional Treatment with Epigallocatechin-3-Gallate, Thymoquinone, and Tannic Acid on the LNCaP Cell Line," Biomedical Sciences Instrumentation, Vol. 42, 2006, pp. 357-362.

[27] N. Chehl, G. Chipitsyna, Q. Gong, et al., "Anti-Inflammatory Effects of the Nigella Sativa Seed Extract, Thzymoquinone, in Pancreatic Cancer Cells," HPB (Oxford), Vol. 11, 2009, pp. 373-381. doi:10.1111/j.1477-2574.2009.00059.x

[28] L. R. Richards, P. Jones, H. Benghuzzi, et al., "A Comparison of the Morphological Changes Associated with Conventional and Sustained Treatment with Pigallocatechin3gallate, Thymoquinone, and Tannic Acid on LNCaP Cells," Biomedical Sciences Instrumentation, Vol. 44 2008, pp. 465-470.

[29] V. B. Astler and F. A. Coller, "The Prognostic Significance of Direct Extension of Carcinoma of the Colon and Rectum," Annals of Surgery, Vol. 139, No. 6, 1954, p. 846.

[30] A. M. Shoieb, M. Elgayyar, P. S. Dudrick, et al., "In Vitro Inhibition of Growth and Induction of Apoptosis in Cancer Cell Lines by Thymoquinone," International Journal of Oncology, Vol. 22, No. 1, 2003, pp. 107-113.

[31] H. Gali-Muhtasib, M. Diab-Assaf, C. Boltze, et al., "Thymoquinone Extracted from Black Seed Triggers Apoptotic Cell Death in Human Colorectal Cancer Cells via a p53-Dependent Mechanism," International Journal of Oncology, Vol. 25, No. 4, 2004, pp. 857-866.

[32] D. R. Worthen, O. A. Ghosheh and P. A. Crooks, "The in Vitro Anti-Tumor Activity of Some Crude and Purified Components of Blackseed, Nigella sativa L.," Anticancer Research, Vol. 18, No. 3A, 1998, pp. 1527-1532.

[33] H. U. Gali-Muhtasib, W. G. Abou Kheir, L. A. Kheir, et al., "Molecular Pathway for Thymoquinone-Induced Cell-Cycle Arrest and Apoptosis in Neoplastic Keratinocytes," Anticancer Drugs, Vol. 15, No. 4, 2004, pp. 389 399. doi:10.1097/00001813-200404000-00012

[34] M. Roepke, A. Diestel, K. Bajbouj, et al., "Lack of p53 Augments Thymoquinone-Induced Apoptosis and Caspase Activation in Human Osteosarcoma Cells," Cancer Biology \& Therapy, Vol. 6, No. 2, 2007, pp. 160-169. 
[35] A. O. Kaseb, K. Chinnakannu, D. Chen, A. Sivanandam, S. Tejwani, M. Menon, et al., "Androgen Receptor and E2F-1 Targeted Thymoquinone Therapy for HormoneRefractory Prostate Cancer," Cancer Research, Vol. 67, No. 16, 2007, pp. 7782-7788. doi:10.1158/0008-5472.CAN-07-1483

[36] T. Yi, S. G. Cho, Z. Yi, X. Pang, M. Rodriguez, Y. Wang, et al., "Thymoquinone Inhibits Tumor Angiogenesis and Tumor Growth through Suppressing AKT and Extracellular Signal-Regulated Kinase Signaling Pathways," Molecular Cancer Therapeutics, Vol. 7, No. 7, 2008, pp. 1789-1796.

[37] S. Banerjee, A. O. Kaseb, Z. Wang, D. Kong, M. Mohammad, S. Padhye, et al., "Antitumor Activity of Gemcitabine and Oxaliplatin Is Augmented by Thymoquinone in Pancreatic Cancer," Cancer Research, Vol. 69, No. 13, 2009, pp. 5575-5583.

[38] S. Attoub, O. Sperandio, H. Raza, K. Arafat, et al., "Thymoquinone as an Anticancer Agent: Evidence from Inhibition of Cancer Cells Viability and Invasion in Vitro and Tumor Growth in Vivo," Fundamental \& Clinical Pharmacology, 2012. doi:10.1111/j.1472-8206.2012.01056.x

[39] T. Yi, S. G. Cho, Z. Yi, X. Pang, et al., "Thymoquinone Inhibits Tumor Angiogenesis and Tumor Growth through Suppressing AKT and ERK Signaling Pathways," Molecular Cancer Therapeutics, Vol. 7, No. 7, 2008, pp. 1789-1796. doi:10.1158/1535-7163.MCT-08-0124

[40] P. A. Hall, D. A. Levinson and A. L. Woods, "Proliferating Cell Nuclear Antigen (PCNA) Immunolocalization in Paraffin Sections: An Index of Cell Proliferation with Evidence of Deregulated Expression in Some Neoplasm," Journal of Pathology, Vol. 162, No. 4, 1990, pp. 285-294.

[41] Y. K. Wang, X. L. Ji, Y. G. Gu, et al., "P53 and PCNA Expression in Glandular Dilatation of Gastric Mucosa," China National Journal of New Gastroenterology, Vol. 2, No. 2, 1996, pp. 106-108.

[42] X. Q. Zhang, "Progress of Study on Suppresser, EGFR and PCNA in Colorectal Cancer," Chinese Journal of New Gastroenterology, Vol. 4, 1996, pp. 327-328.

[43] Y. Zheng, P. M. Kramer, G. Olson, et al., "Prevention by Retinoids of Azoxymethane Induced Tumors and Aberrant Crypt Foci and Their Modulation of Cell Proliferation in the Colon of Rats," Carcinogenesis, Vol. 18, No. 11, 1997, pp. 2119-2125.

[44] W. J. Adams, J. A. Lawson, S. E. Nicholson, et al., "The Growth of Carcinogen Induced Colon Cancer in Rats Is Inhibited by Cimetidine," European Journal of Surgical Oncology, Vol. 19, No. 4, 1993, pp. 332-335.

[45] X. D. Jia and C. Han, "Chemoprevention of Tea on Colorectal Cancer Induced by Dimethylhydrazine in Wistar Rats," World Journal of Gastroenterology, Vol. 6, No. 5, 2000, pp. 699-703.

[46] B. Shpitz, Y. Bomstein, Y. Mekori, et al., "Proliferating Nuclear Antigen as a Marker of Cell Kinetics in Aberrant Crypt Foci Hyperplastic Polyps, Adenomas, and Adenocarcinomas of the Human Colon," American Journal of Surgery, Vol. 174, No. 4, 1997, pp. 425-430. doi:10.1016/S0002-9610(97)00122-0
[47] G. Cohen, R. Mustafi, A. Chumsangsri, et al., "Epidermal Growth Factor Receptor Signaling Is Up-Regulated in Human Colonic Aberrant Crypt Foci," Cancer Research, Vol. 66, No. 11, 2006, pp. 5656-5664. doi:10.1158/0008-5472.CAN-05-0308

[48] D. Longo, "HARRISON'S Hematology and Oncology," McGraw Hill, New York, 2010, 325 p.

[49] J. Folkman, "What Is the Evidence That Tumors Are Angiogenesis Dependent?" Journal of the National Cancer Institute, Vol. 82, No. 1, 1990, pp. 4-6. doi:10.1093/jnci/82.1.4

[50] L. A. Liotta, J. Kleinerman and G. M. Saidel, "Quantitative Relationships of Intravascular Tumor Cells, Tumor Vessels, and Pulmonary Metastases Following Tumor Implantation," Cancer Research, Vol. 34, 1974, pp. 9971004.

[51] G. Gasparini, R. Longo, M. Toi and N. Ferrara, "Angiogenic Inhibitors: A New Therapeutic Strategy in Oncology," Nature Clinical Practice Oncology, Vol. 2, 2005, pp. 562-577. doi:10.1038/ncponc0342

[52] M. M. Cooney, W. Van Heeckeren, S. Bhakta, et al., "Drug Insight: Vascular Disrupting Agents and Angiogenesis-Novel Approaches for Drug Delivery," Nature Clinical Practice Oncology, Vol. 3, 2006, pp. 682-692. doi:10.1038/ncponc0663

[53] G. Bergers and L. E. Benjamin, "Tumorigenesis and the Angiogenic Switch," Nature Reviews Cancer, Vol. 3, No. 6, 2003, pp. 401-410. doi:10.1038/nrc1093

[54] D. J. Kerr, "Targeting Angiogenesis in Cancer: Clinical Development of Bevacizumab," Nature Clinical Practice Oncology, Vol. 1, 2004, pp. 39-43. doi:10.1038/ncponc0026

[55] H. N. Fernando and H. I. Hurwitz, "Targeted Therapy of Colorectal Cancer: Clinical Experience with Bevacizumab," Oncologist, Vol. 9, Suppl. 1, 2004, pp. 11-18. doi:10.1634/theoncologist.9-suppl 1-11

[56] G. Des Guetz, B. Uzzan, P. Nicolas, et al., "Microvessel Density and VEGF Expression Are Prognostic Factors in Colorectal Cancer. Meta-Analysis of the Literature," British Journal of Cancer, Vol. 94, No. 12, 2006, pp. 1823-1832. doi:10.1038/sj.bjc.6603176

[57] N. Ferrara, "Vascular Endothelial Growth Factor: Basic Science and Clinical Progress," Endocrine Reviews, Vol. 25, No. 4, 2004, pp. 581-611.

[58] N. Ferrara, "Molecular and Biological Properties of Vascular Endothelial Growth Factor," Journal of Molecular Medicine, Vol. 77, No. 7, 1999, pp. 527-543. doi:10.1007/s001099900019

[59] Y. Takahashi, Y. Kitadai, C. D. Bucana, et al., "Expression of Vascular Endothelial Growth Factor and Its Receptor, KDR, Correlates with Vascularity, Metastasis, and Proliferation of Human Colon Cancer," Cancer Research, Vol. 55, No. 18, 1995, pp. 3964-3968.

[60] K. Werther, I. J. Christensen, N. Brunner and H. J. Nielsen, "Soluble Vascular Endothelial Growth Factor Levels in Patients with Primary Colorectal Carcinoma, The Danish RANX05 Colorectal Cancer Study Group," European 
Journal of Surgical Oncology, Vol. 26, No. 7, 2000, pp. 657-662. doi:10.1053/ejso.2000.0977

[61] K. Werther, I. J. Christensen and H. J. Nielsen, "The Association between Preoperative Concentration of Soluble Vascular Endothelial Growth Factor, Perioperative Blood Transfusion, and Survival in Patients with Primary Colorectal Cancer," European Journal of Surgery, Vol. 167, No. 4, 2001, pp. 287-292. doi:10.1080/110241501300091480

[62] K. Werther, I. J. Christensen and H. J. Nielsen, "Prognostic Impact of Matched Preoperative Plasma and Serum VEGF in Patients with Primary Colorectal Carcinoma," British Journal of Cancer, Vol. 86, No. 3, 2002, pp. 417423. doi:10.1038/sj.bjc. 6600075

[63] W. S. Tsai, C. R. Changchien, C. Y. Yeh, et al., "Preoperative Plasma Vascular Endothelial Growth Factor but Not Nitrite Is a Useful Complementary Tumor Marker in Patients with Colorectal Cancer," Diseases of the Colon \& Rectum, Vol. 49, No. 6, 2006, pp. 883-894.

[64] F. De Vita, M. Orditura, E. Lieto, et al., "Elevated Perioperative Serum Vascular Endothelial Growth Factor Levels in Patients with Colon Carcinoma," Cancer, Vol. 100, No. 2, 2004, pp. 270-278.

[65] G. Galizia, E. Lieto, F. Ferraraccio, et al., "Determination of Molecular Marker Expression Can Predict Outcome in
Colon Carcinomas," Clinical Cancer Research, Vol. 10, 2004, pp. 3490-3499. doi:10.1158/1078-0432.CCR-0960-03

[66] K. F. Chin, J. Greenman, E. Gardiner, et al., "Pre-Operative Serum Vascular Endothelial Growth Factor Can Select Patients for Adjuvant Treatment after Curative Resection in Colorectal Cancer," British Journal of Cancer, Vol. 83, No. 11, 2000, pp. 1425-1431.

[67] O. Kemik, S. Kemik Ahu, A. Sümer, et al., "Preoperative Vascular Endothelial Growth Factor Levels as a Prognostic Marker for Stage II or III Colorectal Cancer Patients," Cancer Growth and Metastasis, Vol. 4, 2011, pp. 25-32. doi:10.4137/CGM.S7113

[68] G. L. Semenza, "Targeting HIF-1 for Cancer Therapy," Nature Reviews Cancer, Vol. 3, 2003, pp. 721-732. doi:10.1038/nrc1187

[69] J. D. Gordan and M. C. Simon, "Hypoxia-Inducible Factors: Central Regulators of the Tumor Phenotype," Current Opinion in Genetics \& Development, Vol. 17, No. 1, 2007, pp. 71-77. doi:10.1016/j.gde.2006.12.006

[70] M. Erdurmus, R. Yagci, B. Yilmaz, et al., "Inhibitory Effects of Topical Thymoquinone on Corneal Neovascularization," Cornea, Vol. 26, No. 6, 2007, pp. 715-719. doi:10.1097/ICO.0b013e31804f5a45 